\title{
Influencing the oscillation properties of a control system
}

\section{A. Weinmann OVE}

The operation of shifting the poles to the left in the complex plane is transferred to the method of reducing the main oscillation frequency of a control system. Influencing the oscillation properties is realized in an unstructured manner without using solution details of the characteristic equation. The holistic design procedures using the trace of the inverse coefficient matrix and the Michailow distance are applied to find a gradient-based algorithm for improving the controller, after having executed a double-turning or a single-turning and shifting of the closed-loop coefficient matrix.

Keywords: turning the spectrum; selective influence on the oscillation behavior; unstructured operations intentionally

\section{Beeinflussung der Schwingungseigenschaften eines Regelungssystems.}

Die Operation, die Pole in der komplexen Ebene nach links zu verschieben, wird auf eine Methode übertragen, die Schwingungsfrequenz zu reduzieren. Der Einfluss auf das Schwingungsverhalten wird besorgt, ohne detaillierte Strukturen zu verlangen und ohne die charakteristische Gleichung näher zu untersuchen. Der holistische Entwurfsvorgang benützt die Spur der inversen Koeffizientenmatrix oder die Michailow-Distanz, um ein Gradientenverfahren für die Verbesserung des Reglers zu entwickeln, nachdem die Koeffizientenmatrix des Regelkreises in zweifacher oder einfacher Weise gedreht und verschoben wurde.

Schlüsselwörter: Drehung des Spektrums; selektiver Einfluss auf das Schwingungsverhalten; Maßnahmen ohne nähere strukturelle Erfordernis

\section{Motivation. Introduction}

When designing a control system or a general dynamical system, there is often the main interest in influencing the oscillation properties in a global manner, only, or to get an indicator for such an operation at least.

Consider a plant with roughly estimated parameters, e.g. in an agricultural application, and an arbitrarily chosen controller providing a stable but enthusiastically oscillating system. You only want to get less vivid oscillation. With respect to the highly unknown or uncertain plant it is not well-aimed to guess eigenvalue allocation or to select weighting matrices for improving an index of performance. The question also arises where the objective parameters should be taken from

Motivation arises from the interest to improve or change the oscillation properties of a control system as separated as possible by finding a gradient of the current controller; even though the direction obeying the controller to follow the gradient is an additional task. The restriction has to be taken into consideration that current local data of the system are incomplete.

Holistic control system design [2] is an attempt for improving the dynamics of a control system, a method which could also be termed as an unstructured design. Emphasis is put on an action without needing specialized setpoints (e.g. for pole allocation) and without solving a high-order algebraic equation. Primarily, an attack on the oscillation properties is executed without needing help from any eigenvalue assignment.

Using the trace of the inverse of the closed-loop coefficient matrix there are many advantages [2]. By applying the sum of eigenvalues the imaginary parts are cancelled because in physically realizable systems the eigenvalues $\lambda$ are always complex conjugates. Even by using the inverse of the eigenvalues, the imaginary parts do no more play a primary role in the design. In the sum of all eigenvalues $\sum \lambda_{i}[\mathbf{A}]=\operatorname{tr}[\mathbf{A}]=\sum a_{i} \pm j b_{i}=2 a_{i}$ the imaginary parts of the eigenvalues are cancelled at all. In the sum of inverse eigenvalues $\sum 1 /\left(a_{i} \pm j b_{j}\right)$ the $b_{i}$ are conserved partly in $\sum 2 a_{i} /\left(a_{i}^{2}+b_{i}^{2}\right)$.

Repetition for a new approach, that is, $\lambda_{j}[j \mathbf{A}]$, yields the same results in principle only multiplied by $j$.

The Michailow distance [3] is another opportunity for using a holistic approach to improve the dynamics behavior. Consider the determinant of the inverse of the resolvent matrix, i.e., the characteristic polynomial $p_{c l}\left(j \omega, \mathbf{A}_{c \mid}\right) \triangleq \operatorname{det}\left(j \omega \mathbf{I}-\mathbf{A}_{c \mid}\right)$ for $s=j \omega$. It must not include the origin, and should avoid the origin with a distance $h_{0}$, where $h_{0}$ is kept high (or maximized) in modulus.

Supposing the plant and the closed-loop system in state space, with its matrices $\mathbf{A}, \mathbf{A}_{c l} \in \mathcal{R}^{n \times n} ; \mathbf{B} \in \mathcal{R}^{n \times m} ; \mathbf{C}=\mathbf{I}_{n} ; \mathbf{D}=\mathbf{0}$ and the state controller $\mathbf{K} \in \mathcal{R}^{m \times n}$, one has the coefficient matrix of the closed-loop system $\mathbf{A}_{C l}=\mathbf{A}+\mathbf{B K}$. (For a general dynamical system, $\mathbf{A}, \mathbf{K}$ is the fixed and variable part, respectively.)

The starting point of this paper is a mapping operation corresponding to replacing the complex variable $s$ by $\pm j$ s and by $\pm j s+g$, or to replace $\mathbf{A}_{c l}$ by $\mp j \mathbf{A}_{c l}+g \mathbf{l}$.

The derivations are carried out in frequency domain. To illustrate the results in time domain as well, discrete-time system examples are chosen.

\section{The author is Life Senior Member IEEE.}

Weinmann, Alexander, Vienna University of Technology, Institute of Automation and Control, Gusshausstrasse 27-29/376, A-1040 Vienna, Austria

(E-mail: weinmann@acin.tuwien.ac.at) 


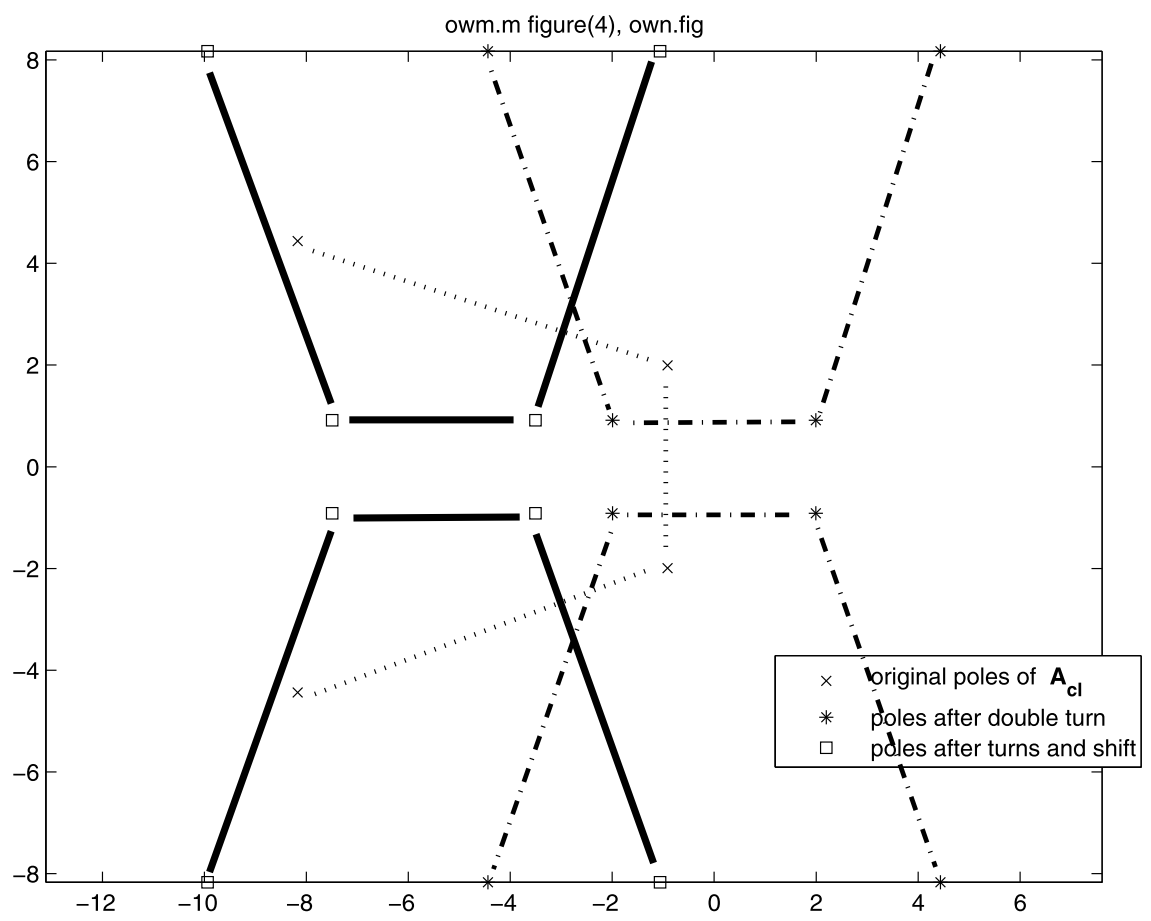

Fig. 1. Turning and shifting the poles (Example 2)

\section{Trace-based influence on the oscillating part.}

\section{Continuous-time system}

Preassume a stable closed-loop system, $\Re e\left\{\lambda_{i}[\mathbf{A}+\mathbf{B K}]\right\}<0 \forall i$, even $\Re e\left\{1 / \lambda_{i}[\mathbf{A}+\mathbf{B K}]\right\}<0 \forall i$. Then its sum is a negative real number as well.

Omitting particular weights and without defining a specified index of performance including the actuation effort, optimal dynamics is expected for shifting all the eigenvalues to the left as much as possible, that is, one has to minimize the following positive-valued expression of eigenvalues $\lambda$ and the trace tr

$$
-\sum_{i} \frac{1}{\lambda_{i}[\mathbf{A}+\mathbf{B K}]}=-\operatorname{tr}\left[\mathbf{A}_{c l}^{-1}\right] \rightarrow \min _{\mathbf{K}}
$$

The closer a pole is located to the imaginary axis the bigger the inverse of the pole, and the more influence on the sum in Eq. (1).

Based on the relation $\partial \operatorname{tr}\left[\mathbf{M}^{-1}\right] / \partial f=-\operatorname{tr}\left[\mathbf{M}^{-2} \partial \mathbf{M} / \partial f\right]$, the gradient of $-\operatorname{tr}\left[\mathbf{A}_{c l}^{-1}\right]$ with respect to $\mathbf{K}$ and $\mathbf{A}_{c l}$ is proportional $-\left[\mathbf{A}_{c l}^{-2} \mathbf{B}\right]^{T}$ and $-\mathbf{A}_{c l}^{-2 T}$, respectively, [2] (see also Eq. (34)).

The main idea of this paper is to put the imaginary parts of the conjugate-complex poles into the role of the real part when influencing the oscillation dynamics is required. Simply replacing $s$ by $j s$ replaces the real-world system with positive constants into polynomials or transfer functions with imaginary constants (see poles after turn in Fig. 1), touching similar results as in [5]. Stability criteria, e.g. Routh or Hurwitz, cannot be applied immediately. Besides, the turn yields a new system where the imaginary parts produce zeros of the characteristic polynomial in the right half-plane. ${ }^{1}$

${ }^{1}$ Shift and rotate: Consider a polynomial $p(s) \operatorname{or} \operatorname{det}(s \mathbf{I}-\mathbf{A})$ and replace $s$ by $j$ s, then the zeros are turned by 90 degrees in clockwise direction. Replacing $\mathbf{A}$ by $j \mathbf{A}$, the eigenvalues are turned in counterclockwise direction. If $\mathbf{A}$ is replaced by $\mathbf{A}+g \mathbf{l}(g>0)$ (or $s:=s-g$ ), the eigenvalues are shifted to the right. Replacing $s$ by $s+g$, a shift to the left by $g$ is performed. Although these relations are very simple they might be got mixed up.
Now, another new system is created. Focussing on the imaginary part of the closed-loop eigenvalues, a turn by 90 degrees is performed, first, and by -90 degrees, second, by replacing $\mathbf{A}_{c l}$ by $\pm j \mathbf{A}_{c l}$, respectively. These systems are switched into series thus defining a new hypothetic system $\mathbf{A}_{f}$ of double order $2 n$

$$
\begin{aligned}
p_{f}(s) & \triangleq \operatorname{det}\left(s \mathbf{I}_{n}-j \mathbf{A}_{c \mid}\right) \times \operatorname{det}\left[\left(s \mathbf{I}_{n}-\left(-j \mathbf{A}_{c}\right)\right]\right. \\
& =\operatorname{det}\left(s^{2} \mathbf{I}_{n}+\mathbf{A}_{c \prime}^{2}\right) \triangleq \operatorname{det}\left(s \mathbf{I}_{2 n}-\mathbf{A}_{f}\right) .
\end{aligned}
$$

In addition, each system is shifted to the left by $g(g>0)$

$$
\begin{aligned}
p_{f}(s) \triangleq & \operatorname{det}\left(s \mathbf{I}_{n}-j \mathbf{A}_{c l}+g \mathbf{I}_{n}\right) \\
& \times \operatorname{det}\left[\left(s \mathbf{I}_{n}+j \mathbf{A}_{c l}\right)+g \mathbf{I}_{n}\right] \\
= & \operatorname{det}\left[(s+g)^{2} \mathbf{I}_{n}+\mathbf{A}_{c l}^{2}\right] \triangleq \operatorname{det}\left(s \mathbf{I}_{2 n}-\mathbf{A}_{f}\right) .
\end{aligned}
$$

The factor $g$ usually is an estimate of the maximum imaginary part of the eigenvalues

$$
g \triangleq \max _{i}\left|\Im m \lambda_{i}\left[\mathbf{A}_{c}\right]\right| .
$$

The system $\mathbf{A}_{f}$ is characterized by eigenvalues symmetric to the real axis. It is suitable for the Routh stability criterion, e.g., of the hypothetic system.

We consider a canonical form $\mathbf{A}_{f}$ the last row of which is termed $\mathbf{f}^{T}$. Its coefficients $f_{i}$ can easily be found by differentiation and equating $s=0$

$$
\left.f_{1}=-\operatorname{det}\left[(s+g)^{2} \mathbf{I}_{n}+\mathbf{A}_{c)}^{2}\right)\right]\left.\right|_{s=0^{\prime}}
$$

and for $i=[1,2 n-1]$

$$
\begin{aligned}
f_{i+1} & \left.=-\frac{1}{i !}\left[\frac{\partial^{i}}{\partial s^{i}} \operatorname{det}\left[(s+g)^{2} \mathbf{I}_{n}+\mathbf{A}_{c l}^{2}\right)\right]\right]_{s=0} \\
& \triangleq-\frac{1}{i !}\left[\frac{\partial^{i}}{\partial s^{i}} \operatorname{det}\left[s \mathbf{I}_{2 n}-\mathbf{A}_{f}\right]\right]_{s=0},
\end{aligned}
$$




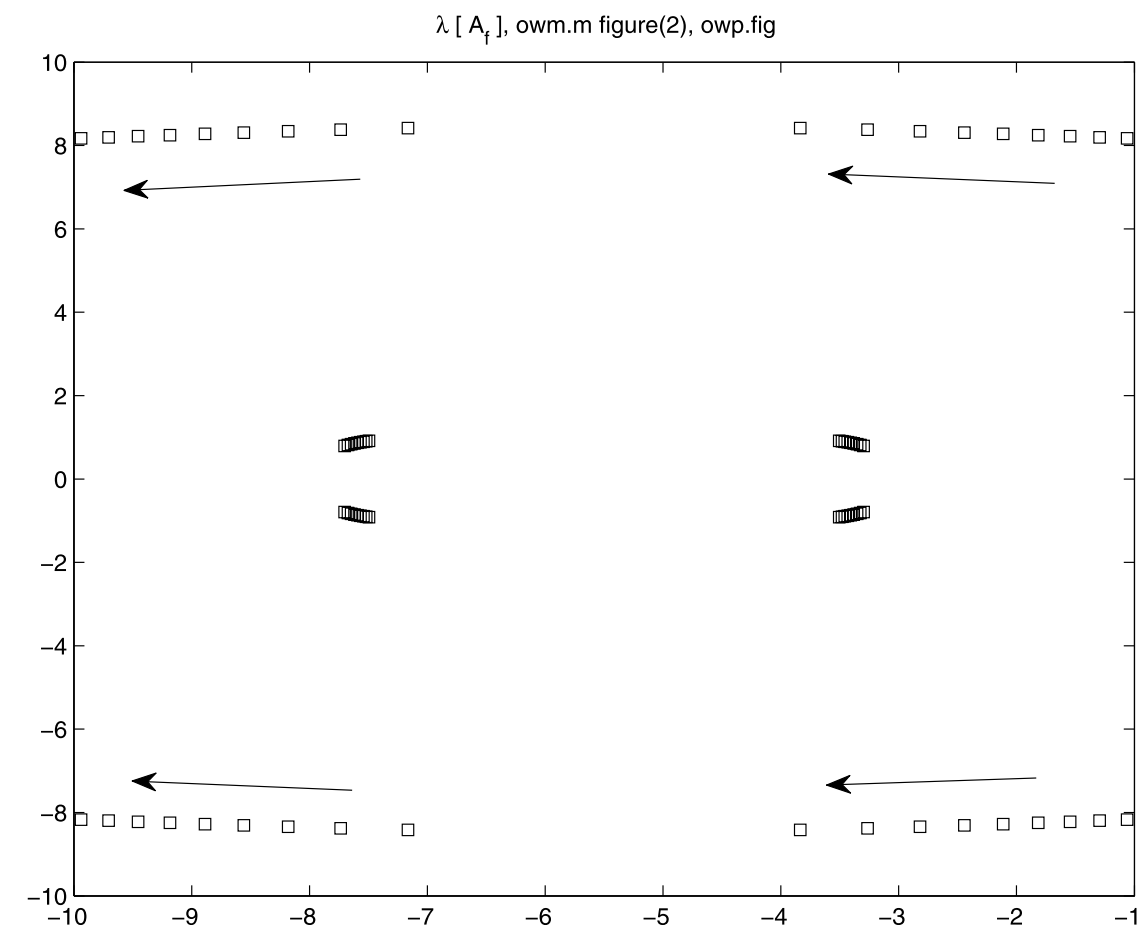

Fig. 2. Eigenvalues of $A_{f}$ of the hypothetic double-turned system during the 9 gradients steps (Example 2)

$$
\mathbf{A}_{f} \triangleq\left(\begin{array}{ccc}
\mathbf{o}_{2 n-1} & & \mathbf{I}_{2 n-1} \\
& \mathbf{f}^{T}
\end{array}\right) \in \mathcal{R}^{2 n \times 2 n} .
$$

Example 1 Consider the simple second-order system $n=2, a_{1}=$ $-5, a_{2}=-2, \lambda_{i}\left[\mathbf{A}_{c l}\right]=-1 \pm j 2$

$$
\begin{aligned}
& \mathbf{A}_{c l}=\left(\begin{array}{cc}
0 & 1 \\
a_{1} & a_{2}
\end{array}\right), \quad \mathbf{A}_{c l}^{2}=\left(\begin{array}{cc}
a_{1} & a_{2} \\
a_{1} a_{2} & a_{1}+a_{2}^{2}
\end{array}\right) \\
& \mathbf{f} \triangleq\left(\begin{array}{llll}
f_{1} & f_{2} & f_{3} & f_{4}
\end{array}\right)^{T}, \\
& p_{f}(s)=\operatorname{det}\left(s \mathbf{I}_{2 n}-\mathbf{A}_{f}\right)=s^{2 n}-\sum_{i=1}^{2 n} f_{i} s^{i-1} \\
& =s^{4}-f_{4} s^{3}-f_{3} s^{2}-f_{2} s-f_{1} .
\end{aligned}
$$

With an additional shift $g$, one has

$$
\begin{aligned}
p_{f}(s) \triangleq & \operatorname{det}\left[(s+g)^{2} \mathbf{I}_{2}+\mathbf{A}_{c l}^{2}\right] \triangleq \operatorname{det}\left(s \mathbf{I}_{4}-\mathbf{A}_{f}\right) \\
f_{1} & =-g^{4}-\left(2 a_{1}+a_{2}^{2}\right) g^{2}-a_{1}^{2} \\
f_{2} & =-2 g\left(2 g^{2}+2 a_{1}+a_{2}^{2}\right) \\
f_{3} & =-\left(6 g^{2}+2 a_{1}+a_{2}^{2}\right) \\
f_{4} & =-4 g .
\end{aligned}
$$

For $g=2$ one finds $\lambda_{j}\left[\mathbf{A}_{f}\right]=-4 \pm j ; 0 \pm j$ from $p_{f}(s)=s^{4}+8 s^{3}+$ $18 s^{2}+8 s+17=0$ at the stability border.

Example 2 Consider a fourth-order continuous-time control system with two conjugate-complex poles $-0.91 \pm j 1.99,-8.16 \pm j 4.43$, $g=5.5$ estimated.

Via turning by $\alpha= \pm \pi / 2$ and shifting by $g$ (see Fig. 1) these quantities can be estimated using Routh or Michailow stability criterion.
Establishing $\mathbf{A}_{f}$, the real $g$ is used a little bit larger than the estimated one, in order to keep $\mathbf{A}_{f}$ stable. Otherwise the gradient of $\operatorname{tr}\left[\mathbf{A}^{-1} \mathbf{B}\right]$ does not operate, or in specific situations, only. In Fig. 1, the corresponding eigenvalues are connected by lines for the sake of better orientation.

There is a remarkable difference between oscillation influence (this paper) and main damping influence [2]. Returning by $\pm \pi / 2$ and reshifting by $-g$ cannot be applied directly. When applying oscillation gradients the double turned eigenvalues are shifted to the left, and the more shifted by gradients the closer they are located to the imaginary axis. The result is that returning would yield eigenvalues which are no more exactly conjugate complex. In spite of this, the changes $\Delta \mathbf{K}$ can be utilized regularly, see Eq. (34).

Changing the imaginary part of the original system, i.e., the real parts of the eigenvalues of the system after turn, the damping performance is also changed to a certain extent. "The polynomial laws retain a drawback".

What are the main advantages using the proposed method with the trace of the inverse of the system matrix? One could suggest to solve the problem of oscillation frequency reduction via classical eigenvalue allocation. Using a full state controller would permit arbitrary eigenvalue assignment. Troubles arise for partial state controller implementation, then the assignment of eigenvalues must be specifically interdependent. Analytical solutions usually fail for the order higher than two

The trace method puts hands on all the available controller parameters $k_{i}$ at once. The reduction progress can be observed via gradient steps, see Fig. 2. It must be observed carefully as well as the setup for the step size. In addition, one has to supervise if the eigenvalue sensitivity is high enough to follow the gradient as intended. The eigenvalue sensitivity is given by operations using eigenvectors $\mathbf{a}_{i}$, $\mathbf{a}_{i}^{\triangleleft}[1,4]$

$$
\frac{\partial \lambda_{i}\left[\mathbf{A}_{c l}\right]}{\partial \mathbf{K}}=\left(\mathbf{I}_{m} \otimes \mathbf{a}_{i}^{\triangleleft * T}\right) \frac{\partial \mathbf{A}_{c l}}{\partial \mathbf{K}}\left(\mathbf{I}_{n} \otimes \mathbf{a}_{i}\right) .
$$




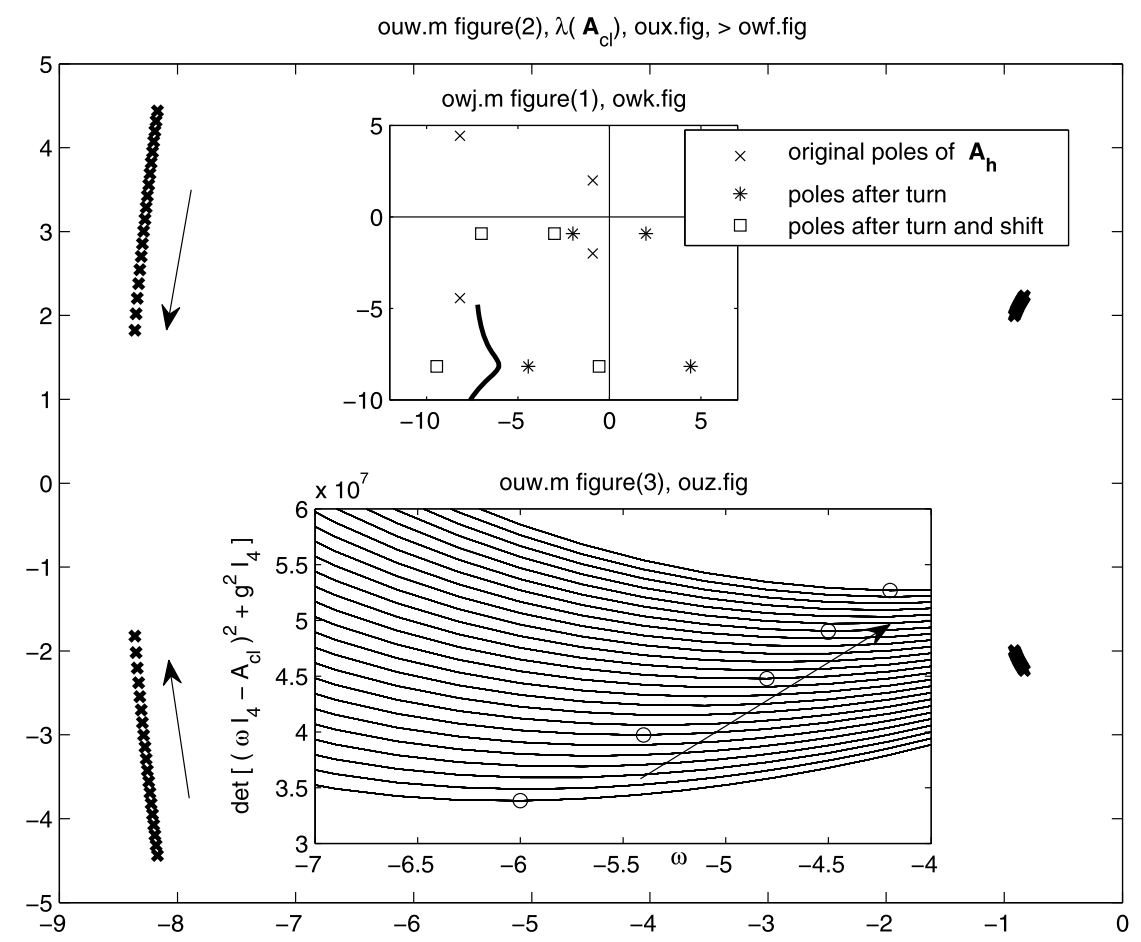

Fig. 3. Reduction of the main $\Im m \lambda_{i}\left[\mathrm{~A}_{c l}\right]$, pole operations (upper subplot, $\left.\mathrm{A}_{h}=\mathrm{A}_{c l}\right)$ and $h_{0 D}^{2}(\omega)$ during 25 gradient steps (lower subplot) for Example 3

In principle, the method in this chapter would also operate by selecting $j \mathbf{A}_{C l}$ alone without the multiplication in Eq. (3) since the gradient results from the squared expression $\left(j \mathbf{A}_{c}\right)^{-2}$.

\section{Influence based on the Michailow characteristic}

The Michailow characteristic results from the characteristic polynomial of the closed-loop control system $p_{C l}\left(s, \mathbf{A}_{C l}\right)$ for $s$ being intentionally replaced by $j \omega$. Intersection of $p_{c l}(j \omega)$ with the origin determines the stability border, i.e., a steady-state oscillation of the closed loop with frequency $\omega$, if all the other eigenvalues are located in the left s-plane. The minimum geometrical distance $h_{0}$ of $p_{c l}\left(j \omega, \mathbf{A}_{c l}\right)$ to the origin at frequency $\omega_{0}$ is an adequate measure of the stability margin. The minimum distance $h_{0}$ squared is

$$
h_{0}^{2}=\operatorname{det}\left(\omega_{0}^{2} \mathbf{I}_{n}+\mathbf{A}_{c)}^{2}\right) .
$$

Increasing $h_{0}^{2}$ usually improves the stability quality. The poles are shifted to the left primarily, in an unstructured manner. This is also true in the opposite direction by lowering $h_{0}^{2}$.

The gradient or differential sensitivity of $h_{0}^{2}$ with respect to $\mathbf{K}_{y}$ is used to improve stability in an optimal way [3].

Now, if $\mathbf{A}_{c l}$ is turned to $j \mathbf{A}_{c l}$, the unstructured relation takes place between $h_{O D}^{2}$ and the real parts of the hypothetic turned system, i.e. the imaginary part of the original system. We replace $\mathbf{A}_{C l}$ by $j \mathbf{A}_{C l}$ in the well-known Michailow characteristic, turning counterclockwise and shifting to the left by $g$. Then a complex characteristic of the hypothetic system results

$$
\begin{aligned}
h_{0}(\omega) & \triangleq\left|p_{C l, D}\left(s, j \mathbf{A}_{C l}\right)\right|_{s=j \omega} \mid \\
& =\left|\operatorname{det}\left[s \mathbf{I}_{n}-j \mathbf{A}_{c l}+g \mathbf{I}_{n}\right]\right|_{s=j \omega} \mid .
\end{aligned}
$$

Using the complex conjugate, the square of the absolute value is

$$
\begin{aligned}
h_{O D}^{2}(\omega) \triangleq & \operatorname{det}\left(j \omega \mathbf{I}_{n}-j \mathbf{A}_{c l}+g \mathbf{I}_{n}\right) \\
& \times \operatorname{det}\left(-j \omega \mathbf{I}_{n}+j \mathbf{A}_{c l}+g \mathbf{I}_{n}\right) \\
= & \operatorname{det}\left[\left(\omega \mathbf{I}_{n}-\mathbf{A}_{c l}\right)^{2}+g^{2} \mathbf{I}_{n}\right] .
\end{aligned}
$$

We find $\min _{\omega}$ at $\omega_{0}$ by equating $\partial\left(h_{0 D}^{2}\right) / \partial \omega=0$. Equating $h_{0 D}^{2}=0$ as well yields $g$ and $\omega_{0}$. Only an estimate of $g$ at the stability border is required.

Example 3 In Fig. 3 (with the setup in th upper subplot) the original pole $-8.1+j 4.4$, after having turned and shifted, is closest to the imaginary axis and dominantly influences the characteristic $h_{0 D}^{2}(\omega)$. Increasing the minimum distance to the origin [3] forces the turned poles to the left. This is equivalent to reducing the imaginary value of the original pole.

The result is illustrated in Fig. 3. Reduction of the oscillation is demonstrated. In the upper subplot, the original poles are depicted, and the turned and shifted as well. In addition, a small part of $h_{0 D}^{2}(\omega)$, with $\omega$-axis vertically, is included to demonstrate that the peak of the characteristic is at -8.1 . The lower subplot shows the turned Michailow characteristic $h_{0 D}^{2}(\omega)$ in the course of running 25 gradient steps. The characteristics increase upwards while executing the steps of $h_{0 D}^{2}$ of Eq. (25). The shift parameter $g$ is included and used for estimating the largest imaginary value of $\mathbf{A}_{c /}$.

\section{Discrete-time system}

Establishing a discrete-time system with sampling interval $T$ is performed by using the basic continuous-time system and the generalized eigenvalue $\operatorname{det}(\lambda[\mathbf{A}] \mathbf{Q}-\mathbf{A})=0$ to enter the $z$ and $w$ domain. Via MATLAB: $[V, D]=\operatorname{eig}(A, Q)$. The operations are performed in $w$ domain in similar manner as in the previous sections.

$$
\operatorname{det}\left(z \mathbf{I}_{n}-e^{\mathbf{A} T}\right)=\operatorname{det}\left(\frac{1+w T / 2}{1-w T / 2} \mathbf{I}_{n}-e^{\mathbf{A} T}\right)=0
$$



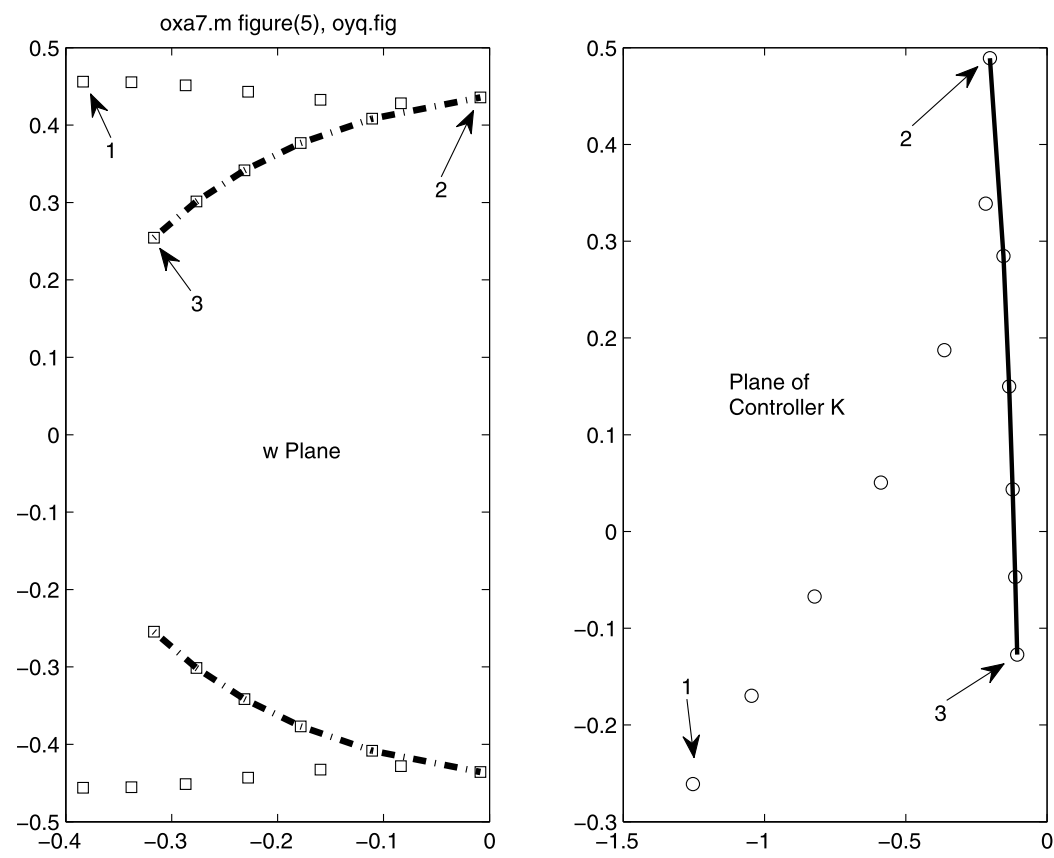

Fig. 4. Step changes of the $w$ eigenvalues (left) and the controller K components (right) (Example 4)

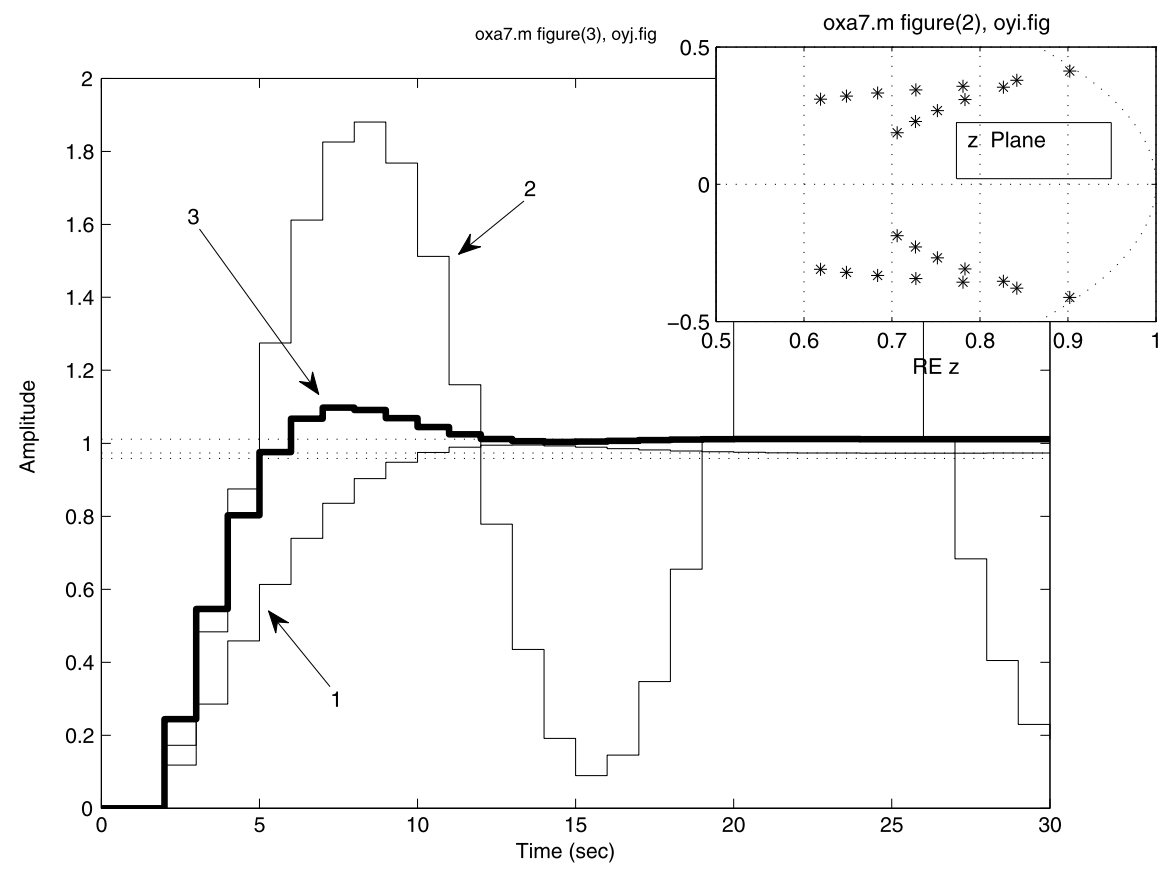

Fig. 5. Resulting influence on the step response (Example 4)

$$
\begin{array}{r}
\operatorname{det}\left(w\left(\mathbf{I}_{n}+e^{\mathbf{A} T}\right) T / 2+\mathbf{I}_{n}-e^{\mathbf{A} T}\right)=0 \\
\lambda_{w}=\operatorname{eig}(\operatorname{expm}(A T)-\operatorname{eye}(n), Q) \text { (in MATLAB) }
\end{array}
$$

where $\mathbf{Q}=\left(\mathbf{I}_{n}+e^{\mathbf{A} T}\right) T / 2$. The controller $\mathbf{K}$ is operating the system following the gradient increments $\Delta \mathbf{K}$ of the appendix.

The mapping $w(z)$ can be selected with or without inclusion of $T / 2$. The system target also might be executing the sampling period $T$ dependent on the deviation. That is, there is a multiple influence on estimating the shift parameter $g$ and on spreading the inverse eigenvalues, see Eqs. (1) and (8)

Example 4 For a second-order SISO system, the discrete-time equivalent with $m=1$ is selected. Assume, the initial controller operates the system near the unit circle stability boarder (Point 2 in Fig. 4 and Fig. 5). Following the gradient steps leads to point 3. For comparison purpose, the method [2] is demonstrated for gradient steps from point 1 to 2 which would cause a stability loss. 


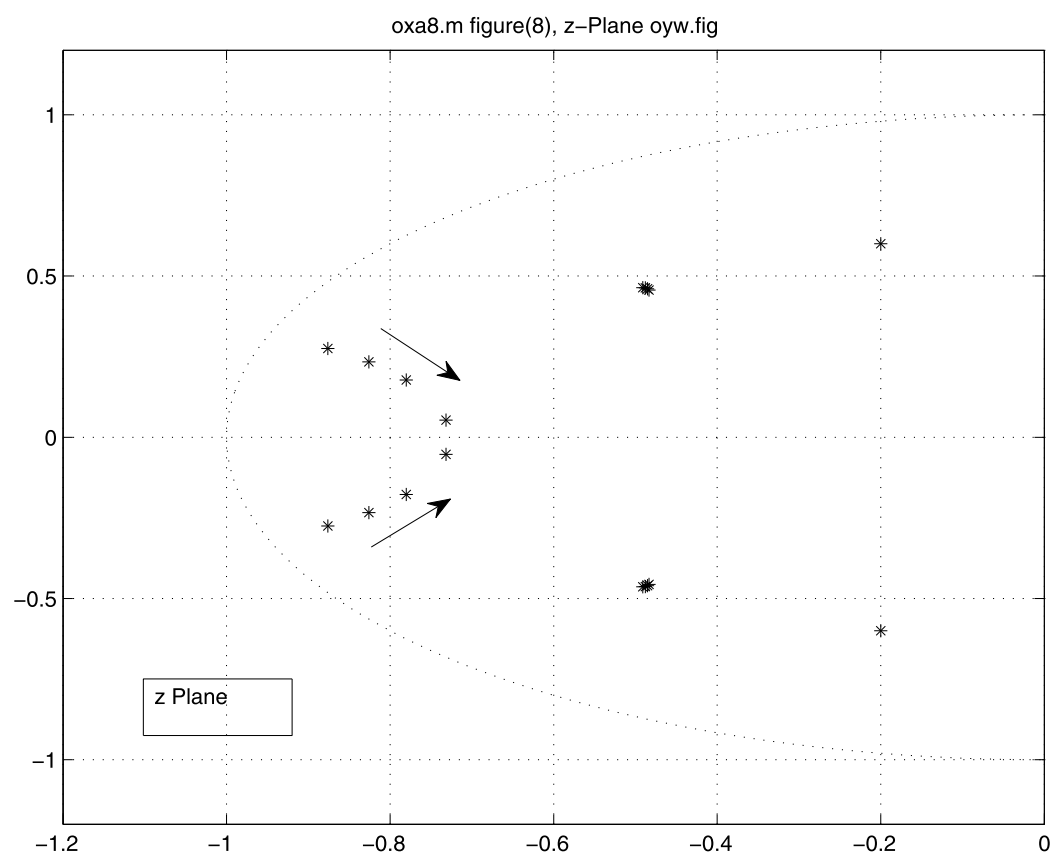

Fig. 6. Progress in the z-plane (Example 5)

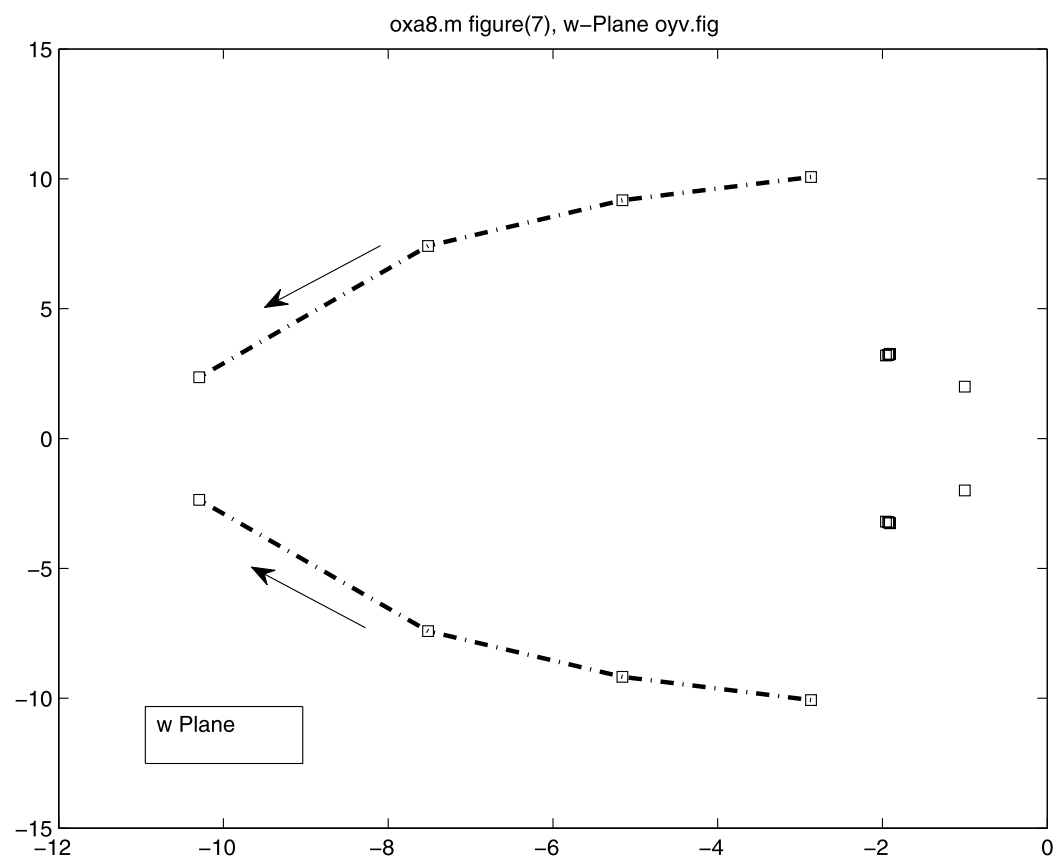

Fig. 7. Progress in the w-plane (Example 5)

Example 5 In a system of 6th order, a distinct attack on the eigenvalue with maximum imaginary component is executed with three steps. In Figs. 6 and 7 the progress in z-plane and w-plane is demonstrated via three steps selective with respect to the eigenvalue with the highest frequency. The arrows indicate the reduction of oscillation components. In Fig. 8 the change in discrete-time domain is reported.

\section{Outlook and conclusion}

The main goal of this paper is to change the oscillation properties without need of external setup parameters such as used in eigen- value allocation. Two methods are presented, aiming at primarily reducing the oscillation properties of a linear time-invarying control system. The methods do not require structured setup and setpoints for the design.

However, there is still a lot to do concerning several analytical proofs, regions of operation, multiple turns, e.g. $e^{j \alpha} \mathbf{A}_{c l}$ and shifting, where $\operatorname{det}\left[s^{2} \mathbf{I}_{n}-2 s \cos \alpha+\mathbf{A}^{2}\right]$ results, or $\operatorname{det}\left[(s-h)^{2} \mathbf{I}_{n}+\left(\mathbf{A}-g \mathbf{I}_{n}\right)^{2}\right]$, or $\operatorname{det}\left[s^{2} \mathbf{I}_{n}+\left(\mathbf{A}-g \mathbf{I}_{n}\right)^{2}\right]$ by shifting before turning. One-dimensional search versus $\alpha$ to check stability border is an opportunity. The smaller $\alpha$ the more problematic the $90^{\circ}$ turn is. Selected action on the coupled eigenvalues is problematic initially. It is still open if it 


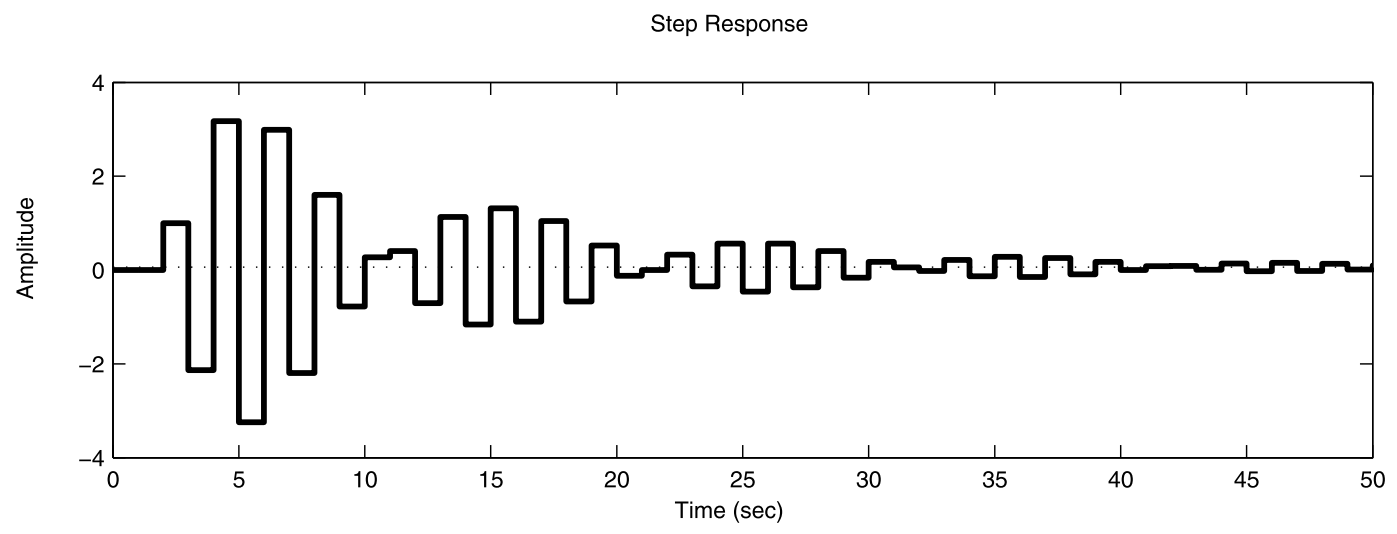

oxa8.m figure(9), dstep oyx.fig

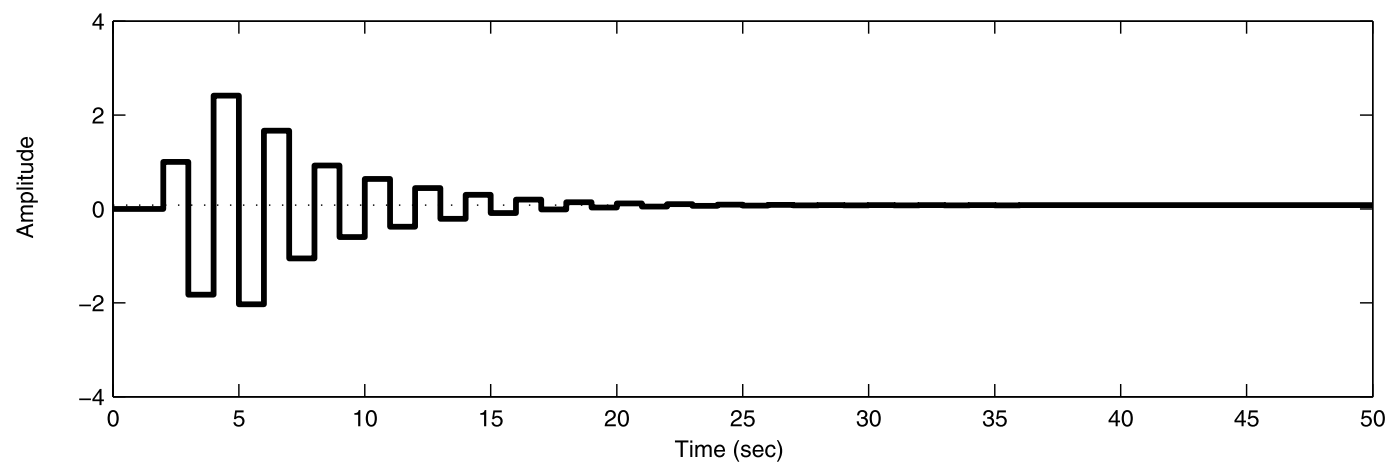

Fig. 8. Discrete-time step response in Example 5 before and after three steps (upper and lower subplot)

is worth while to take the double order of the trace-based method into account. The Michailow characteristic method does not require double order but uses a hypothetic imaginary system to establish the gradient procedure. An opportunity is to combine the methods of influencing damping and oscillation, alternately.

There are examples where a selective attack on the eigenvalues with maximum imaginary component is executed. In general, examples show that the expected operations can be put into action successfully, even though no guarantee for a perfect operation exists when opposite to eigenvalue sensitivity.

\section{Acknowledgements}

Open access funding provided by TU Wien (TUW).

Open Access This article is distributed under the terms of the Creative Commons Attribution 4.0 International License (http://creativecommons.org/ licenses/by/4.0/), which permits unrestricted use, distribution, and reproduction in any medium, provided you give appropriate credit to the original author(s) and the source, provide a link to the Creative Commons license, and indicate if changes were made.

\section{Appendix}

The question is which increment $\Delta \mathbf{K}$ yields an increment $\Delta \mathbf{A}_{f}$ of the holistic setup

$$
\begin{aligned}
& \operatorname{det}\left[(s+g)^{2} \mathbf{I}_{n}+(\mathbf{A}+\mathbf{B K})^{2}\right]=\operatorname{det}\left(s \mathbf{I}_{2 n}-\mathbf{A}_{f}\right), \\
& \operatorname{det}\left[(s+g)^{2} \mathbf{I}_{n}+(\mathbf{A}+\mathbf{B K}+\mathbf{B} \Delta \mathbf{K})^{2}\right] \\
& \quad=\operatorname{det}\left(s \mathbf{I}_{2 n}-\mathbf{A}_{f}-\Delta \mathbf{A}_{f}\right) .
\end{aligned}
$$

Based on the correspondences [4]

$$
\begin{aligned}
& \operatorname{col}(\mathbf{H F G}) \equiv\left(\mathbf{G}^{T} \otimes \mathbf{H}\right) \operatorname{col} \mathbf{F}, \\
& \left.\operatorname{det}(\mathbf{A}+\Delta \mathbf{A})\right|_{\|\Delta \mathbf{A}\|_{F} \ll 1} \\
\doteq & \operatorname{det} \mathbf{A}+\left[\operatorname{col}\left(\operatorname{adj} \mathbf{A}^{T}\right)\right]^{T} \cdot \operatorname{col} \Delta \mathbf{A},
\end{aligned}
$$

Equation (5) with $\mathbf{A}_{c l}=\mathbf{A}+\mathbf{B K}$ is applied in $w$ and $z$ domain. Omitting intermediate calculations, one finds the resulting $\Delta \mathbf{K}$

$$
\mathbf{h}_{1}^{T} \operatorname{col} \Delta \mathbf{K}=\mathbf{h}_{r}^{T} \operatorname{col} \Delta \mathbf{A}_{f}
$$

where $\Delta \mathbf{A}_{f} \propto \mathbf{A}_{f}^{-2 T}[2]$ and the abbreviations

$$
\begin{gathered}
\mathbf{A}_{h} \triangleq(s+g)^{2} \mathbf{I}_{n}+(\mathbf{A}+\mathbf{B K})^{2}, \\
\mathbf{h}_{1}^{T} \triangleq 2\left[\operatorname{col}\left(\operatorname{adj} \mathbf{A}_{h}^{T}\right)\right]^{T}\left[(\mathbf{A}+\mathbf{B K})^{T} \otimes \mathbf{B}\right], \\
\mathbf{h}_{r} \triangleq-\operatorname{col}\left[\operatorname{adj}\left(\mathbf{I}_{2 n}-\mathbf{A}_{f}\right)^{T}\right.
\end{gathered}
$$

have been used. The components of $\Delta \mathbf{K}$ are combined with $\mathbf{h}_{1}$ in a scalar product, i.e. there is a multitude of solutions to fulfil the scalar product of the right side of the final equation. Minimum square result, pseudo inverse, or any other actuation signal limitation can be implemented. 
Transferring a matrix into a vector concatenating all the columns is performed by the operator col, adj is the adjoint matrix $\operatorname{adj}(\mathbf{M})=$ $\mathbf{M}^{-1}$. detM. The comparison of the coefficients in Eq. (30) can be executed for any numeric $s$ if $\Delta \mathbf{K}$ is very small in norm sense.

\section{References}

1. Feliachi, A. (1986): Decentralized stabilization of interconnected systems. Int. J. Control, 44, 1499-1505.

\section{Author}

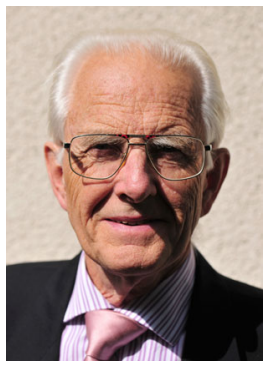

\section{Alexander Weinmann}

Born in Vienna, Austria, in 1937, 1960 Dipl.Ing., 1962 Dr. techn., 1966 Habilitation "Elektrische Antriebs- und Regelungstechnik", 1968-2005 O. Univ.-Prof. "Elektrische Regelungs- und Steuerungstechnik" at Vienna University of Technology. Head of the Institute of Automatic Control 1968-2004. Long-term Dean of the Faculty of Electrical Engineering, co-initiating the foundation. Managing head of the former computing center of the entire university, chair of several commissions for academic administration,
2. Weinmann, A. (2001): Control system design based on holistic eigenvalue allocation. E\&I, Elektrotech. Inf.tech., 118, 167-173.

3. Weinmann, A. (2005): A dialog-oriented and gradient-based stability margin in uncertain systems. Cybern. Syst.: Internat. J., 36(7), 641-666.

4. Weinmann, A. (2012): Performance gradients in automatic control analysis. A catalogue of correspondences. Int. J. Automat. Austria, 20, 1-100. http://www.acin.tuwien ac.at/de/Publikationen/Zeitschriften/IJAA. Second edition.

5. Wu, A. G., Zhang, Y. (2017): Complex conjugate matrix equations for systems and control. Singapore: Springer.

tuition and curriculum development.

He worked on various topics related to optimal control systems and design, identification, robustness, observers. Single author (without co-authorship) of roughly 140 scientific papers and seven books in several editions. 18 patents.

Consultant of ELIN with actual project responsibility, and other enterprises, up to 2006

Editor and associate editor of several journals. Honory member OVE and ASCS. Among others: Awards Karoline and Guido Krafft, Kardinal Innitzer, Golden Stefan Medal of OVE. 